\title{
Bortezomib synergizes with nocodazole in p53 mediated DNA damage response signalling in A549 lung cancer cells
}

\author{
Gülşah ALBAYRAK ${ }^{*}$ (1) \\ 1 Department of Medical Biology, Faculty of Medicine, Ufuk University, Ankara, Turkey. \\ * Corresponding Author. E-mail albayrakgulsah@gmail.com (G.A.).
}

Received: 08 October 2020 / Revised: 12 April 2021/ Accepted: 18 April 2021

\begin{abstract}
The adoption of new treatment modalities is required to improve outcomes in lung cancer treatment as lung cancer has lowest survival rates, along with liver and pancreatic cancer. Bortezomib is a proteasome inhibitor that has higher anticancer effect in combination therapies. The aim of this study was to investigate whether bortezomib could have additional anticancer effect with antineoplastic tubulin binding agent -nocodazole in A549 lung cancer cells. Apoptosis related gene expression levels of Noxa, Bcl-xL, Casp3 and Casp7 were measured by real-time PCR after treatment with $30 \mathrm{nM}$ bortezomib, $0.3 \mu \mathrm{g} / \mathrm{ml}$ nocodazole and with their combination for 24 hours. Synergistic effect on DNA damage response was investigated at protein levels by checking p53 and cleaved PARP expressions. Induction of apoptotis was determined at protein expression level by western blotting of XIAP, Bcl-X and Bim. It was found that nocodazole combined bortezomib treatment induced apoptosis via p53 mediated DNA damage response signalling. P53 and cleaved PARP protein expressions were increased significantly after combination treatment. Apoptosis related genes Noxa, Casp3 and Casp7 mRNA expressions were elevated significantly after combination treatment. This study concludes that bortezomib potentiates the effect of nocodazole via DNA damage induced apoptosis in A549 lung cancer cells.
\end{abstract}

KEYWORDS: Bortezomib; Nocodazole; DNA damage; lung cancer.

\section{INTRODUCTION}

Lung cancer is one of the leading cause of cancer related deaths worldwide [1]. Survival rates are lower as disease is generally diagnosed at an advanced stage [2]. Therefore, the adoption of new treatment modalities like the new drugs and combination therapies is required to improve outcomes in lung cancer treatment.

Bortezomib is the first FDA approved proteasome inhibitor and clinical trials are ongoing ever since to investigate its anticancer activity in solid tumors [3]. Bortezomib is a reversible $26 \mathrm{~S}$ proteasome inhibitor that degrades proteins in the cell under normal conditions [4]. Inhibition of $26 \mathrm{~S}$ proteasome directs cancer cells to apoptosis [5].

Nocodazole is a synthetic tubulin binding agent with neoplastic activity [6]. Nocodazole binds to betatubulin and interferes with the microtubule dynamics. This prevents mitosis and induces apoptosis in tumor cells [7]. Bortezomib increases the effect of traditional anticancer agents (doxorubicin, cisplatin, paclitaxel, irinotecan and radiation etc. in combinatorial treatment regimes [8]. Therefore, the aim of this study was to investigate whether bortezomib could potentiate the anticancer effect of nocodazole in vitro. Bortezomib's IC50 value was previously identified as $30 \mathrm{nM}$ for A549 cells [9]. Nocodazole is used at $0.1-1 \mu \mathrm{g} / \mathrm{ml}$ concentration range for 12-48 hours [10]. Their synergistic effect in lung cancer cells has not been studied previously. The aim of this research was to investigate the synergic effect of bortezomib and nocodazole in terms of apoptosis in A549 lung cancer cells. Therefore, apoptosis related mRNA expressions (Noxa, Bcl-xL, Casp3 and Casp7) were measured by real time PCR after $30 \mathrm{nM}$ bortezomib, $0.3 \mu \mathrm{g} / \mathrm{ml}$ nocodazole and with their combination treatment on A549 lung cancer cells at 24 hours. In order to shed more light on the molecular mechanism of these drugs; P53, PARP cleavage, Bcl-X, XIAP, Bim protein expression levels were investigated by western blot. 


\section{RESULTS}

\subsection{Bortezomib potentiates nocodazole via 533 regulated DNA damage response signalling pathway}

A549 lung cancer cells were treated with $30 \mathrm{nM}$ bortezomib, $0.3 \mu \mathrm{g} / \mathrm{ml}$ nocodazole and with their combination for $24 \mathrm{~h}$. p53 guards the genome by coordinating a variety of DNA-damage-response (DDR) mechanisms. To investigate the effect of nocodazole and bortezomib on DNA damage response signalling; p53 and cleaved PARP protein levels were measured by western blot at 24 hours. p53 protein expression level was upregulated significantly upon each treatment. PARP cleavage is another indicator of DNA damage. Cleaved PARP protein expression level was also upregulated significantly upon each treatment (Figure 1). It was found that bortezomib potentiated the effect of nocodazole in DNA damage signalling pathway (Figure 2).
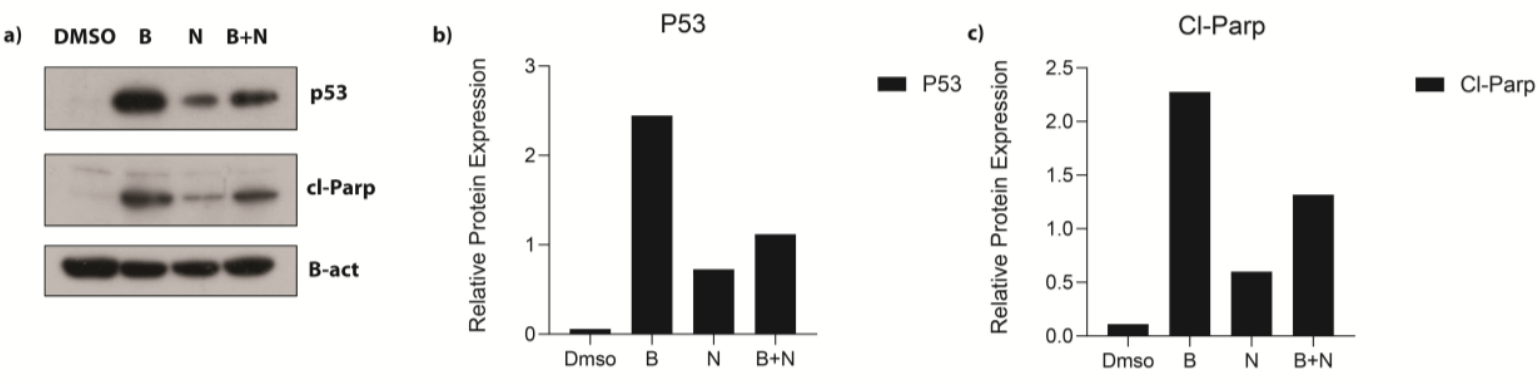

Figure 1. a) A549 cells were treated with indicated concentrations of bortezomib and nocodazole for $24 \mathrm{~h}$. Bortezomib and nocodazole combination increased p53 protein expression in A549 cells. PARP cleavage was detected by western blot analysis upon bortezomib and nocodazole for $24 \mathrm{~h}$. b) Relative protein expressions were represented as fold change for p53 normalized to $\beta$-Actin. c) Relative protein expressions were represented as fold change of the PARP cleavage normalized to $\beta$-Actin. (B: Bortezomib, N: Nocodazole, B+N: Bortezomib and nocodazole combination)

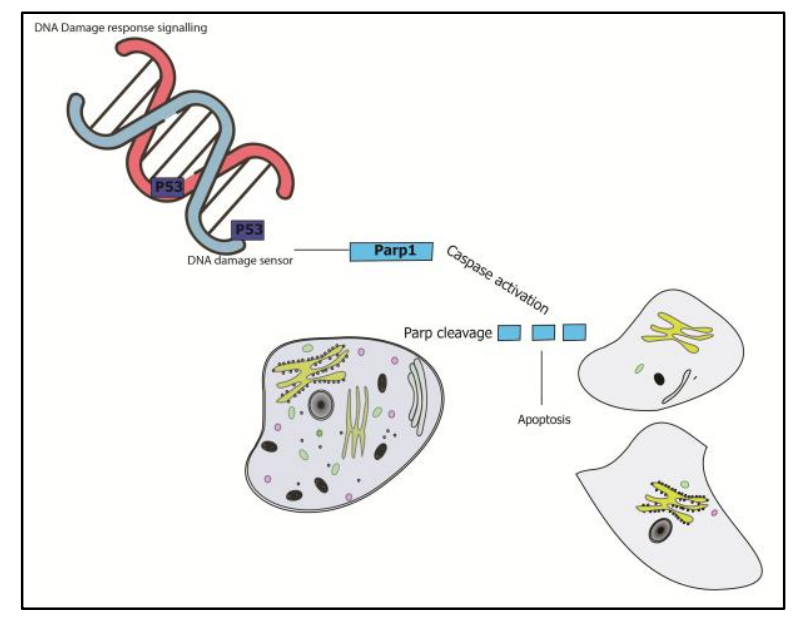

Figure 2. Schematic representation of DNA damage signaling.

\subsection{Bortezomib and nocodazole effects on apoptosis}

Noxa mRNA expression levels were upregulated upon $30 \mathrm{nM}$ Bortezomib and combination; whereas it was decreased with nocodazole treatment (Figure 3a). Bcl-xL is a Bcl-2 protein family member that modulates apoptosis by controlling mitochondrial membrane permeability [11] Single borzetomib treatment induced Bcl-xL upregulation at 24 hours (Figure 3b) Caspases are crucial mediators of apoptosis. Caspase-3 catalyzes the cleavage of many key cellular proteins during apoptosis [12]. Caspase 3 mRNA expression levels were upregulated with all treatment conditions (Figure 3c), whereas Caspase 7 levels were only upregulated by bortezomib treatment (Figure 3d). 

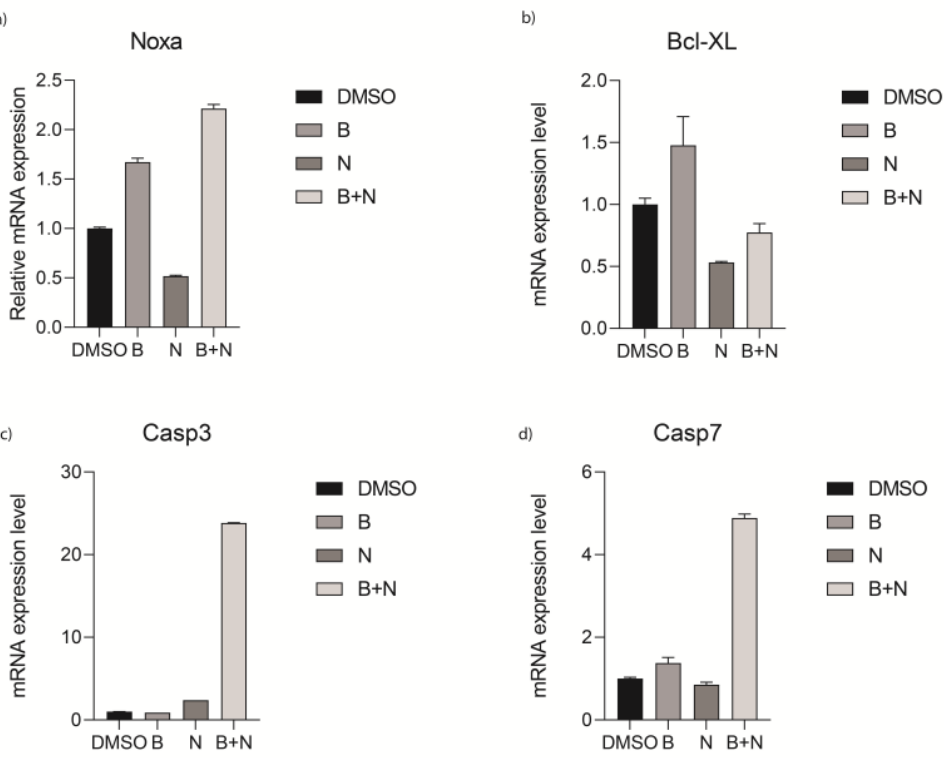

Figure 3. Apoptotic genes were quantified by qPCR. A549 cells were treated with $30 \mathrm{nM}$ bortezomib 0.3 $\mu \mathrm{g} / \mathrm{ml}$ nocodazole and with their combination for $24 \mathrm{~h}$. mRNA expression levels for Noxa, Bcl-xL, Casp-3 and Casp7 were measured by qPCR. mRNA expression values were normalized to the Gapdh internal control (B: Bortezomib, N: Nocodazole, B+N: Bortezomib and nocodazole combination).

In order to check the induction of apoptosis at protein expression level; XIAP, Bcl-X and Bim levels were investigated by western blot. Nocodazole treatment inhibited the X-linked inhibitor of apoptosis protein (XIAP) in single and combination treatment (Figure 4a, 4b). Bcl-X protein expression levels were decreased in each treatment (Figure 4a, 4c). Bim protein expressions were slightly increased upon bortezomib treatment, however it was decreased with nocodazole treatment (Figure 4a, 4d).

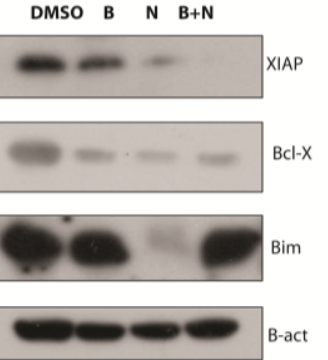

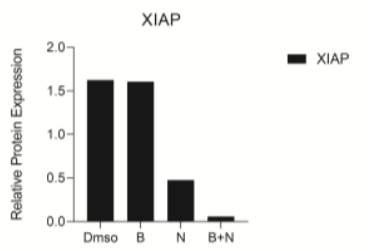

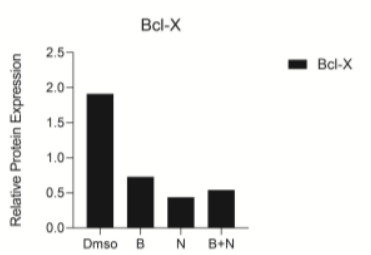

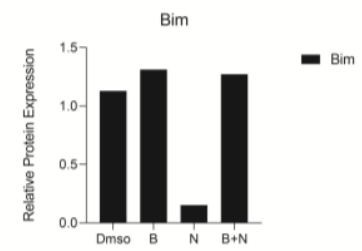

Figure 4. a) A549 cells were treated with indicated concentrations of Bortezomib and Nocodazole for $24 \mathrm{~h}$. and protein expression levels of XIAP, Bcl-X and Bim were checked by western blot. b) Relative protein expressions were represented as fold change for XIAP normalized to $\beta$-Actin. c) Relative protein expressions were represented as fold change of Bcl-X normalized to $\beta$-Actin d) Relative protein expressions were represented as fold change of Bim normalized to $\beta$-Actin. (B: Bortezomib, N:Nocodazole, $\mathrm{B}+\mathrm{N}$ : Bortezomib and nocodazole combination.

\section{DISCUSSION}

Bortezomib is a proteasome inhibitor that has synergic effect when used in combination with the other anticancer drugs [13]. Therefore combination of bortezomib with another antioneoplastic agent nocodazole was investigated in A549 lung cancer cells. p53 acts as a tumor suppressor and reacts to stress signals in different responses. One of the most important p53 function is to induce apoptosis [14]. p53 responds to DNA damage and if DNA damage is not repairable; p53 induces apoptosis [15]. It was found that bortezomib and nocodazole treatment upregulated p53 protein expression level significantly. 
Poly(ADP-ribose) polymerase PARP- 1 is a $113 \mathrm{kDa}$ molecule that is cleaved to 89 and $24 \mathrm{kDa}$ fragments during the apoptosis. Cleavage of PARP-1 by caspases is considered as a hallmark of apoptosis [16,17]. Bortezomib and nocodazole treatments increased the levels of PARP cleavage significantly and this result is consistent with the p53 protein expression. Increasing cleaved PARP levels upon combination treatment suggests that more DNA damage were accumulated when cells were treated with the proteasome inhibitor. Microtubule active drugs like nocodazole is known to cause mitotic arrest followed by apoptosis [18]. It was found that nocodazole and its combination with bortezomib induced Noxa, Casp3 and Casp7 activation at mRNA level. XIAP overexpression is found to be promoting bladder cancer invasion in vitro and lung metastasis in vivo [19]. In another study XIAP over-expression was identified as a poor prognostic marker in breast cancer [20]. Nocodazole and its combination with bortezomid inhibited XIAP protein expression therefore that might be the another underlying mechanism that direct cells into apoptosis. Targeting apoptosis in cancer treatment is highly promising as cancer cells loss the balance between cell division and cell death. In this study, it was found that nocodazole leveraged the anti-cancer effect of bortezomib by triggering DNA damage sensing mechanisms in A549 lung cancer cells. This apoptosis mechanism might be wired in other types of cancer cells as well.

\section{CONCLUSION}

Although this study is limited with a single cell line in vitro, the results demonstrate that bortezomib and nocodazole have synergic anticancer effect on A549 cells via triggering DNA damage induced apoptosis.

\section{MATERIALS and METHODS}

\subsection{Cell culture and chemicals}

A549 cells were provided from ATCC (\#CCL-185, American Type Culture Collection, Manassas, VA) and grown in DMEM medium supplemented with 10\% Fetal bovine serum (FBS) Cells were grown in an incubator in $5 \% \mathrm{CO}_{2}$ at $37^{\circ} \mathrm{C}$. Nocodazole and bortezomib were supplied from Santa Cruz, Texas, USA. Nocodazole and bortezomib were dissolved in DMSO, the maximum concentration of DMSO was used as a vehicle control. A549 cells were treated with $30 \mathrm{nM}$ Bortezomib, $0.3 \mu \mathrm{g} / \mathrm{ml}$ Nocodazole and with their combinations for 24 hours. Relevant doses were previously identified in the literature $[9,10]$ and that doses were found sufficient to trigger apoptosis.

\subsection{Protein expression profiles by western blot}

Protein lysates were collected after 24 treatment with the drugs and quantified by using the BCA Assay Kit. $20 \mu \mathrm{g}$ of protein lysates were denatured for 5 minutes at $95^{\circ} \mathrm{C}$ in LDS non-reducing sample buffer and loaded onto the 10\% Tris-glycin gels. The gels were transferred to the PVDF membrane at $300 \mathrm{mAmp}$ for 90 minutes. Membranes were blocked with 5\% non-fat milk powder in TBS-T for 1 hour at room temperature and incubated overnight at $4^{\circ} \mathrm{C}$ with the primary antibodies for P53, cleaved PARP, XIAP, Bcl- $x$, Bim and Bactin at 1:1000 dilution (Thermo Pierce, Rockford, IL, USA). Blots were washed with TBS-T subsequently. Protein bands were detected by using the secondary antibody (Thermo Pierce, Rockford, IL, USA) and the blots were visualized by BioVision ECL Western Blotting Substrate Kit (Biovision, California, USA).

\subsection{Gene expression analysis by RT-qPCR}

RNA was isolated by using Trizol reagent (Invitrogen, Thermo Fischer Scientific, USA) 2000 ng total RNA was reverse transcribed by Superscript III cDNA Synthesis Kit (Invitrogen, Thermo Fischer Scientific, USA) Forward and reverse primer sequences were provided in the appendix. 10-20 cycles of specific target amplification was performed by SYBR Green qPCR master mix. RT- qPCR analysis was performed in Roche LightCycler qPCR. Gapdh was used as internal control when calculating cq value. $\Delta \Delta C \mathrm{Cq}$ method was used to quantify the gene expression levels. mRNA expression levels for Noxa, Bcl-xL, Casp3 and Casp7 were measured by qPCR. Data was analyzed and plotted in Graphpad prism software.

\subsection{Statistical analysis}

Experiments were performed in three different replicates. Statistical analysis was performed by using GraphPad Prism software. Differences between the control and treatment groups were compared by $t$ - test. The results were expressed as the mean \pm standard error mean. Western blot image densities were calculated by Imagej and statistical analysis, plotting of the data were performed in GraphPad Prism software. 
Author contributions: Concept - GA.; Design - GA; Materials - GA.; Data Collection and/or Processing - Writing GA.

Conflict of interest statement: The author declares no conflicts of interest. No competing financial interests exist.

\section{REFERENCES}

[1] Barta JA, Powell CA, Wisnivesky JP. Global Epidemiology of Lung Cancer. Ann Glob Health. 2019; 85(1): 8. [CrossRef]

[2] Blandin Knight S, Crosbie PA, Balata H, Chudziak J, Hussell T, Dive C. Progress and prospects of early detection in lung cancer. Open Biol. 2017; 7(9): 170070. [CrossRef]

[3] Chen D, Frezza M, Schmitt S, Kanwar J, Dou QP. Bortezomib as the first proteasome inhibitor anticancer drug: current status and future perspectives. Curr Cancer Drug Targets. 2011; 11(3): 239-53. [CrossRef]

[4] Budenholzer L, Cheng CL, Li Y, Hochstrasser M. Proteasome Structure and Assembly. J Mol Biol. 2017; 429(22): 3500-3524. [CrossRef]

[5] Bonvini P, Zorzi E, Basso G, Rosolen A. Bortezomib-mediated 26 S proteasome inhibition causes cell-cycle arrest and induces apoptosis in CD-30+ anaplastic large cell lymphoma. Leukemia. 2007; 21(4): 838-42. [CrossRef]

[6] National Center for Biotechnology Information (2020). PubChem Compound Summary for CID 4122, Nocodazole. https:// pubchem.ncbi.nlm.nih.gov/compound/Nocodazole (Accessed October 6, 2020).

[7] Lu Y, Chen J, Xiao M, Li W, Miller DD. An overview of tubulin inhibitors that interact with the colchicine binding site. Pharm Res. 2012; 29(11): 2943-71. [CrossRef]

[8] Kapoor P, Ramakrishnan V, Rajkumar SV. Bortezomib combination therapy in multiple myeloma. Semin Hematol. 2012; 49(3): 228-42. [CrossRef]

[9] Taromi S, Lewens F, Arsenic R, Sedding D, Sänger J, Kunze A, Möbs M, Benecke J, Freitag H, Christen F, Kaemmerer D, Lupp A, Heilmann M, Lammert H, Schneider CP, Richter K, Hummel M, Siegmund B, Burger M, Briest F, Grabowski P. Proteasome inhibitor bortezomib enhances the effect of standard chemotherapy in small cell lung cancer. Oncotarget. 2017; 8(57): 97061-97078. [CrossRef]

[10] Nocodazole product data sheet. https://www.cellsignal.co.uk/products/activatorsinhibitors/nocodazole/2190?Ntk=Products\&Ntt=2190 (Accessed October 6, 2020).

[11] Stevens M, Oltean S. Modulation of the Apoptosis Gene Bcl-x Function Through Alternative Splicing. Front Genet. 2019; 10: 804. [CrossRef]

[12] Porter AG, Jänicke RU. Emerging roles of caspase-3 in apoptosis. Cell Death Differ. 1999; 6(2): 99-104. [CrossRef]

[13] Sooman L, Gullbo J, Bergqvist M, Bergström S, Lennartsson J, Ekman S. Synergistic effects of combining proteasome inhibitors with chemotherapeutic drugs in lung cancer cells. BMC Res Notes. 2017; 10(1): 544. [CrossRef]

[14] Fridman JS, Lowe SW. Control of apoptosis by p53. Oncogene. 2003; 22(56): 9030-40. [CrossRef]

[15] Pitolli C, Wang Y, Candi E, Shi Y, Melino G, Amelio I. p53-Mediated Tumor Suppression: DNA-Damage Response and Alternative Mechanisms. Cancers (Basel). 2019; 11(12): 1983. [CrossRef]

[16] Chaitanya GV, Steven AJ, Babu PP. PARP-1 cleavage fragments: signatures of cell-death proteases in neurodegeneration. Cell Commun Signal. 2010; 8: 31. [CrossRef]

[17] Gobeil S, Boucher CC, Nadeau D, Poirier GG. Characterization of the necrotic cleavage of poly(ADP-ribose) polymerase (PARP-1): implication of lysosomal proteases. Cell Death Differ. 2001; 8(6): 588-94. [CrossRef]

[18] Beswick RW, Ambrose HE, Wagner SD. Nocodazole, a microtubule de-polymerising agent, induces apoptosis of chronic lymphocytic leukaemia cells associated with changes in Bcl-2 phosphorylation and expression. Leuk Res. 2006; 30(4): 427-36. [CrossRef]

[19] Yu Y, Jin H, Xu J, Gu J, Li X, Xie Q, Huang H, Li J, Tian Z, Jiang G, Chen C, He F, Wu XR, Huang C. XIAP overexpression promotes bladder cancer invasion in vitro and lung metastasis in vivo via enhancing nucleolinmediated Rho-GDI $\beta$ mRNA stability. Int J Cancer. 2018; 142(10): 2040-2055. [CrossRef]

[20] Hussain AR, Siraj AK, Ahmed M, Bu R, Pratheeshkumar P, Alrashed AM, Qadri Z, Ajarim D, Al-Dayel F, Beg S, AlKuraya KS. XIAP over-expression is an independent poor prognostic marker in Middle Eastern breast cancer and can be targeted to induce efficient apoptosis. BMC Cancer. 2017; 17(1): 640. 